\title{
Farmaci antipertensivi a confronto: costi e benefici per il paziente e la collettività
}

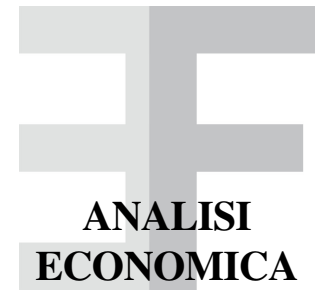

Francesco Vittorio Costa\#

\begin{abstract}
Pharmacoeconomic analysis of antihypertensive treatment should be performed following a correct methodological evaluation and considering with accuracy both costs and benefits of different therapeutic options. Costs evaluation is frequently performed simply examining the retail price of drugs which represents only a part (usually no more than $50 \%$ ) of cumulative costs of therapy.

Controlled clinical trials are the main source of information about benefits of therapy, but probably, in many cases, they underestimate the benefits of treatment and are unable to differentiate the effects of different drugs because of a too short follow-up.

Benefits should be calculated not only in terms of saved lives or prevented events, but also in terms of prevention-regression of target organ damage and of quality of life of patients. If analysis are performed correctly, more recent drugs, like ATII antagonists, even if they have a higher retail price, become highly costeffective thanks to their protective activity against events, and to unbeatable levels of compliance and persistence which are associated with treatment with these drugs.

Comparison between old (cheaper) and newer (more expensive) drugs, and recommendations to start therapy with the cheaper drugs, are a nonsense since to achieve goal blood pressure combinations of more drugs are always necessary.

Treatment of hypertension, if extended to all patients and performed aggressively, must be considered not only a life-saving, but also a money-saving tool.
\end{abstract}

Farmeconomia e percorsi terapeutici 2003; 4 (4): 193-200

\section{PREMESSA}

Nonostante la terapia si sia dimostrata in grado di ridurre sensibilmente le complicanze cardiovascolari dell'ipertensione, ancora oggi solo una piccola percentuale di pazienti viene curata in maniera adeguata e tale da normalizzare la pressione arteriosa. Molti pazienti vengono trattati in maniera insufficiente e molti altri, forse la maggioranza, non ricevono alcuna terapia.

Uno studio recente ha evidenziato come la prevalenza di ipertensione nel nostro paese, nei soggetti adulti (età >35 anni) sia oggi valutabile in circa il 38\%. Negli Stati Uniti, la cui popolazione ha un' età media inferiore alla nostra, la prevalenza d'ipertensione è assai più bassa $(27,8 \%)[1]$.

Lo stesso studio ha anche rilevato come in Italia la percentuale di soggetti che ricevono una terapia antipertensiva (a prescindere dalla sua efficacia nel normalizzare la pressione) sia assai modesta: arriva infatti al 32\%, mentre negli USA supera il $50 \%$. E se si valuta il numero dei pazienti trattati che raggiungono effettivamente un buon controllo pressorio, i dati sono ancora più desolanti.

Il Brisighella Heart Study [2], studio che prevede il controllo periodico dell'intera popolazione di un piccolo comune della Romagna, ha messo in luce come nel corso degli anni, fino al 1992, ci sia stato un progressivo miglioramento del controllo dell'ipertensione, mentre al controllo successivo, nel 1996, non si è osservato un miglioramento, bensì un peggioramento del controllo pressorio della popolazione (Figura 1).

In ogni caso, poco più del $20 \%$ degli ipertesi trattati raggiunge livelli normali di pressione (<140/90 mmHg).

Dato che la frequenza, il tipo e la gravità delle complicanze sono strettamente correlati ai valori della pressione, è facilmente ipotizzabile che un miglior controllo nella popolazione porterebbe ad un'evidente riduzione delle complicanze. Tale obiettivo potrebbe essere raggiunto esclusivamente trattando in modo aggressivo il maggiore numero possibile di pazienti (possibilmente tutti) in modo da normalizzare la pressione a tutti gli ipertesi.
\# Professore Associato in Medicina Interna, Università degli Studi di Bologna 
Perciò sarebbe necessario somministrare combinazioni di farmaci ad una percentuale molto elevata e crescente della popolazione (oltre il $60 \%$ dei soggetti ultrasessantacinquenni è iperteso e l'età media della nostra popolazione è in continuo aumento). La conseguenza sarebbe un inevitabile aumento dei costi immediati.

Si pone quindi il problema: i nuovi farmaci (inibitori dei recettori dell' angiotensina in primis, ma anche calcio-antagonisti e ACE-inibitori), il cui prezzo d'acquisto è più elevato di quello di diuretici e beta-bloccanti, presentano vantaggi tali da giustificarne l'impiego? E il loro utilizzo su larga scala è economicamente sopportabile?

Solo un approccio farmacoeconomico corretto ci può consentire di valutare se tali opzioni siano economicamente sostenibili o se, come da qualche parte si sostiene, la terapia con i farmaci più nuovi debba essere riservata solo a pazienti con caratteristiche particolari, mentre negli altri si deve agire scegliendo sempre i farmaci a basso prezzo.

Occorre tener presente a questo punto che la gestione economica delle terapie viene demandata ad amministratori che perseguono obiettivi di bilancio a breve termine (sei mesi, un anno), mentre nel caso dell'ipertensione, specie nei pazienti a rischio meno elevato, i vantaggi completi di un trattamento efficace si possono valutare solo nel lungo periodo (da non meno di 5 anni fino a 20-30 anni). Si rischia quindi che le strategie di contenimento dei costi terapeutici vengano definite da chi tiene in conto non il benessere dei pazienti, ma i bilanci delle aziende sanitarie.

Senza considerare che una persona che sta bene resta nel circuito produzione-consumi, e

\section{quindi crea risorse invece di consumarle. \\ Questa situazione è di difficile correzione, e solo un medico ben informato può far com- prendere anche a chi non ha cognizioni clini- che che un trattamento efficace risulta alla lun- ga economicamente vantaggioso anche se ri- chiede un investimento iniziale. \\ Percentuale di pazienti in trattamento che raggiunge un buon controllo pressorio $(P A<140 / 90 \mathrm{mmHg})$. Dati dal Brisighella Heart Study [2]}

Nella terapia dell'ipertensione la situazione è particolarmente complessa in quanto esistono numerose opzioni terapeutiche e i prezzi $\mathrm{d}$ 'acquisto dei farmaci differiscono tra loro in maniera significativa. Se non si attuano valutazioni corrette di costi e benefici, si rischia di fare scelte non solo sfavorevoli per i pazienti, ma anche finanziariamente scorrette.

\section{VALUTAZIONEDICOSTIE BENEFICI DELLATERAPIAANTIPERTENSIVA}

Una valutazione farmacoeconomica appropriata può essere effettuata utilizzando una delle quattro tecniche di analisi decisionale tradizionalmente impiegate negli studi di economia sanitaria quando si confrontano alternative diverse sulla base dei relativi costi e benefici: a) minimizzazione dei costi; b) analisi costoefficacia; c) analisi costo-utilità; d) analisi costo-beneficio.

La valutazione dei costi è analoga in tutte questi tecniche, ma la valutazione degli effetti ottenibili con la terapia è differente.

L'analisi minimizzazione dei costi si applica quando vi sia la dimostrazione o la presunzione che le alternative terapeutiche confrontate sono equivalenti. In tal caso il confronto viene attuato unicamente sulla base dei costi e l'opzione terapeutica meno costosa risulta ovviamente quella più conveniente. Partendo dalla considerazione che tutti i farmaci antipertensivi, quando utilizzati singolarmente a dosi adeguate, producono una riduzione dei valori pressori difficilmente differenziabile in termini quantitativi, alcuni decisori sono tentati di adottare la tecnica "minimizzazione dei costi" per decidere quale farmaco antipertensivo sia più conveniente. Questa tecnica, tuttavia, non è adatta per una corretta e robusta valutazione farmacoeconomica dei farmaci antipertensivi attualmente disponibili, dotati di diverso meccanismo d'azione, differente tollerabilità e compliance, e soprattutto di differente potenzialità nel ridurre il rischio cardiovascolare.

L'analisi costo-efficacia e l'analisi costoutilità, che molti autori considerano una variante della prima, si applicano quando le alternative confrontate producono risultati terapeutici equivalenti sul piano qualitativo, ma differenti sul piano quantitativo (ad esempio una diversa percentuale di successi terapeutici indicati come normalizzazione della pressione arteriosa o come anni di vita guadagnati o come anni di vita ponderati per la qualità di vita cioè come QALY). In questo caso l'alternativa più conveniente è quella che presenta un rapporto costo-efficacia o costo-utilità più basso. Poiché spesso nuove terapie sono contemporaneamente più efficaci ma an- 
che più costose di quelle standard di riferimento, l'analisi costo-efficacia utilizza il rapporto costo-efficacia incrementale (ICER) come parametro in base al quale decidere se sia accettabile l'innovazione proposta in relazione alle limitate risorse disponibili.

L'analisi costo-utilità, a differenza dell'analisi costo-efficacia, include nella valutazione dei benefici indotti dal trattamento anche le modificazioni della qualità di vita dei pazienti integrandole con gli eventuali guadagni in quantità di vita. L'unità QALY è diventato uno standard sempre più utilizzato come indicatore di efficacia e utilità nelle valutazioni farmacoeconomiche di terapie croniche dove $\mathrm{i}$ benefici sanitari sono complessi e comportano modificazioni della durata della vita e della qualità di vita dei soggetti trattati. È questo il caso delle varie terapie antipertensive disponibili che, oltre a ridurre o normalizzare la pressione arteriosa, possono comportare risultati sanitari differenti, far guadagnare un numero di anni di vita differente alla popolazione trattata, essere più o meno efficaci nel ridurre il danno d'organo nel prevenire le gravi complicanze cardiovascolari (infarti, ictus, rivascolarizzazioni) e produrre un livello qualitativo di vita più o meno elevato in relazione alla ridotta morbilità e al profilo di tollerabilità dei farmaci usati.

L'analisi costo-beneficio si applica quando le alternative che si intende confrontare producono effetti quantitativi e qualitativi differenti, sicché si rende necessario esprimere gli effetti in unità monetarie piuttosto che in unità fisiche. Questo tipo di analisi non viene normalmente applicata negli studi farmacoeconomici, mentre viene utilizzata prevalentemente dai grandi decisori istituzionali (governi centrali e regionali) per scegliere l'allocazione delle risorse a livello di macrosistemi economici, quando si desideri confrontare programmi di interventi che possono afferire al campo sanitario e a campi extrasanitari (ad esempio una campagna di vaccinazione con un programma di educazione civica).

\section{Valutazione dei costi}

Una valutazione accurata dei costi deve innanzitutto esaminare i costi che non producono alcun beneficio, per poterli eliminare.

Una diagnosi corretta di ipertensione è il primo presupposto per evitare terapie in soggetti che non ne hanno bisogno e che quindi consumano risorse senza trarne beneficio alcuno.

Il controllo pressorio insufficiente comporta spese con vantaggi solo parziali. L'insufficiente controllo della pressione può dipendere dalla scelta non adeguata dei farmaci, da una inaccurata individualizzazione della terapia e soprattutto da una bassa compliance del paziente. La cattiva compliance influisce contempora- neamente sui benefici e sui costi e può essere intesa come un'importante fonte di spesa senza benefici o senza tutti i benefici potenziali attesi.

Vedremo in seguito come tale parametro sia particolarmente rilevante, dato che proprio nel campo della compliance i nuovi farmaci risultano nettamente superiori ai vecchi.

Il costo della terapia si compone di molte voci il cui peso relativo può variare anche di molto da un farmaco ad un altro. Inoltre, alcune voci di costo (ad esempio ospedalizzazioni, perdita di ore di lavoro, perdita di anni di vita) possono diminuire come conseguenza positiva del trattamento e costituire, quindi, un risparmio (costo negativo) che si somma algebricamente agli altri fattori di costo per determinare un costo globale netto.

Bisogna sottolineare che il prezzo d'acquisto dei farmaci, spesso utilizzato come unico parametro per valutare il costo della terapia, è solo una delle voci che compongono i costi globali del trattamento (Tabella 1).

Occorre quindi considerare, oltre al prezzo d'acquisto, il costo delle visite mediche e degli esami di laboratorio, gli eventuali ricoveri in ospedale (una giornata di ricovero può costare come un intero anno di terapia), il tempo perduto dai pazienti, i costi del trasporto per recarsi ai controlli e la perdita d'attività lavorativa e più in generale di produttività.

Altre voci che influiscono pesantemente sui costi, come ricordato in precedenza, sono la compliance e la persistenza al trattamento. I farmaci che garantiscono elevati livelli di compliance e di persistenza (i più nuovi) influiscono meno sui costi finali. Farmaci dal prezzo più basso, ma che richiedono esami o visite mediche più frequenti, o che si associano ad una compliance più bassa e/o a necessità di cambiamenti di terapia, possono alla fine risultare molto più costosi di altri dal prezzo d'acquisto più alto.

L'analisi eseguita qualche anno fa da Hilleman e collaboratori [3], in uno dei pochi studi di confronto del costo di varie classi di

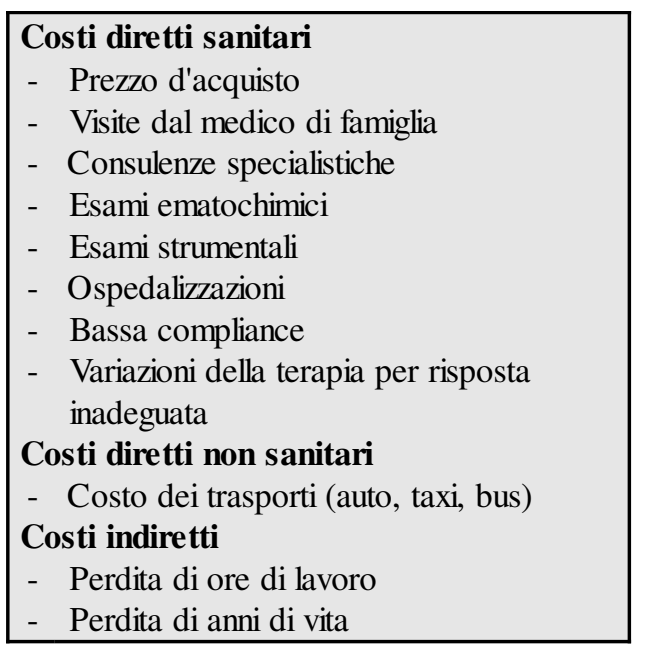

\section{Tabella 1}

Fattori che determinano il costo globale della terapia nella prospettiva della società 


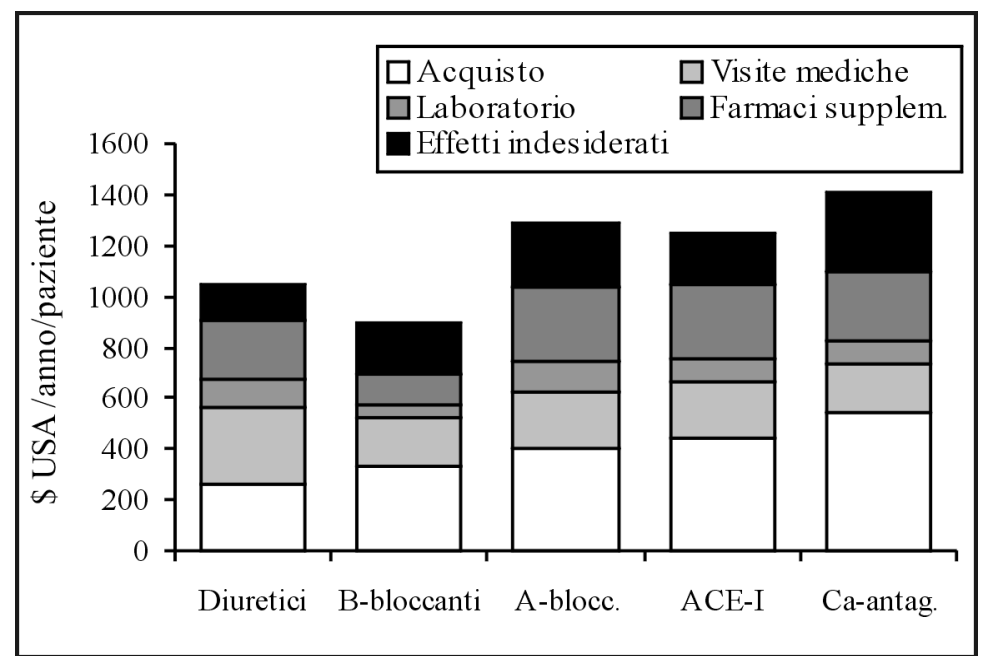

\section{Figura 2}

Costo medio di diverse classi di farmaci usati nell'ipertensione lieve [3] (da Hilleman DE et al, Clin Ther 16, 1994)

\section{Figura 3}

Fattori che contribuiscono al costo dell'ipertensione in Italia - Lo studio GREAT [4] (da FV Costa et al, 1999)

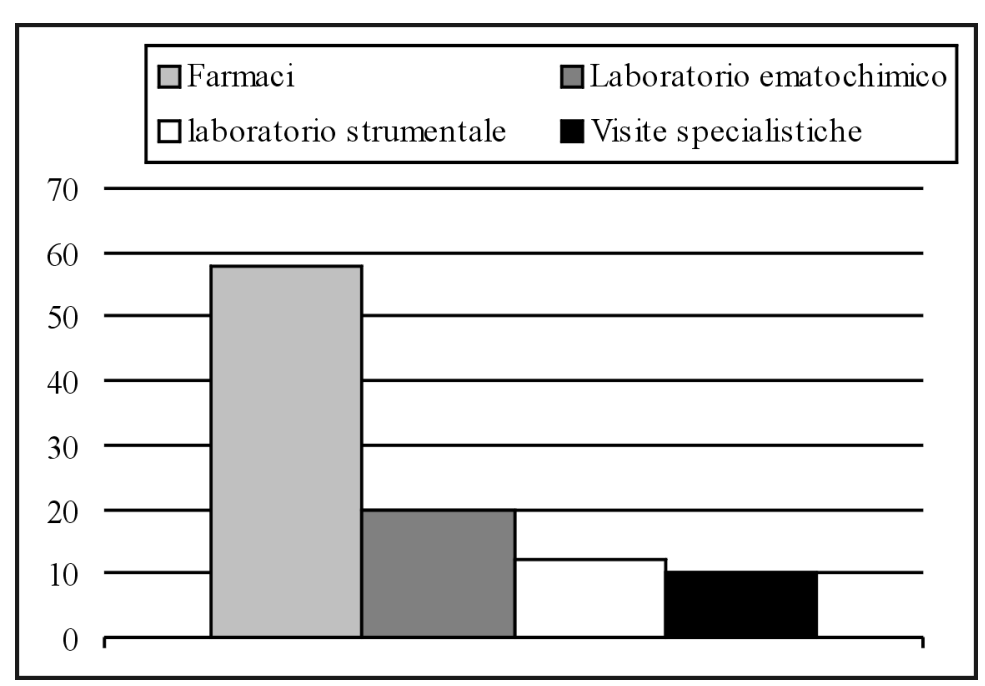

È evidente che qualsiasi terapia, per produrre al meglio i suoi effetti positivi, deve essere assunta con regolarità (compliance) e costanza. La costanza nel tempo (persistenza) è uno dei fattori che differenzia maggiormente i farmaci antipertensivi e assume anche rilevanza economica, poiché è stato evidenziato come i soggetti che cambiano la terapia prescritta affrontino costi annui del $20 \%$ superiori a coloro che non debbono cambiarla [5]. Diviene così importante, sia clinicamente che economicamente, prescrivere farmaci che garantiscono una buona persistenza.

Gli ACE-inibitori e soprattutto gli ATII-antagonisti sono i farmaci che, in tutti gli studi finora pubblicati, si associano ai livelli più elevati di persistenza. Al contrario, i tiazidici, che secondo le conclusioni dello studio ALLHAT [6] dovrebbero essere considerati la prima scelta perché efficaci come gli altri farmaci e più economici, si associano a livelli di persistenza bassissimi. Conlin [7], in una popolazione di pazienti seguita per quattro anni dai medici di famiglia, e che quindi riproduce la realtà clinica di tutti i giorni e non quella artificiale degli studi clinici controllati, ha dimostrato come già dopo sei mesi il $75 \%$ dei pazienti cui era stato prescritto un diuretico l'ha sospeso, e dopo 4 anni solo il 16\% continua la terapia, mentre con ACE-inibitori e, soprattutto, ATII-antagonisti le percentuali di soggetti persistenti sono molto più elevate (Figura 4).

Questa osservazione è stata confermata da altri studi e anche nei nostri pazienti si è visto che, a due anni dall'inizio della terapia, poco più del $40 \%$ di pazienti continua il diuretico, mentre la terapia con ATII-antagonisti viene proseguita da oltre il $70 \%$ dei pazienti (Figura 5).

Queste osservazioni assumono un particolare rilievo quando si consideri che la prima terapia assunta da un paziente ne condiziona la compliance futura. Un paziente che sia soddisfatto del trattamento sarà un paziente compliante e persistente; al contrario, se la prima terapia ha causato problemi, quel paziente sarà sempre restio a eseguire correttamente $\mathrm{e}$ costantemente il trattamento [8].

Ciò dimostra ancora una volta come i dati ricavati dagli studi clinici controllati non siano esportabili in toto alla pratica medica quotidiana, e che farmaci equivalenti quando testati in ambiente sperimentale, alla prova pratica dei fatti diventano del tutto diversi.

Il farmaco migliore del mondo può divenire il peggiore se i pazienti non sono in grado di assumerlo regolarmente. In pratica il medico deve sempre operare in modo che il paziente sia in grado di assumere regolarmente il trattamento, che lo continui nel tempo e che da esso tragga i maggiori vantaggi ottenibili e, se possibile, al prezzo più basso. 


\section{Valutazione dei benefici}

La valutazione dei benefici che qui viene esposta si basa esclusivamente sui risultati degli studi clinici controllati.

A tale proposito occorre ribadire che essi rappresentano una realtà molto diversa dalla pratica clinica. Innanzitutto la durata di tali studi risulta troppo breve, specie nei pazienti appartenenti a classi di rischio lieve o intermedio, per poter evidenziare eventuali differenze tra terapie egualmente capaci di ridurre la pressione [9].

Un follow-up di 4-5 anni è nulla rispetto agli anni di terapia di solito utilizzati in questi pazienti (spesso più di 20 anni). È quindi probabile che eventuali differenze tra terapie non possano essere evidenziate in un periodo di tempo così limitato. Per utilizzare un paragone un po' semplicistico, potremmo dire che se vogliamo sapere qual è il maratoneta più forte, non possiamo basarci sui risultati di una corsa di 5.000 metri. Non solo potremmo non vedere differenze significative, ma addirittura potrebbe avvenire che chi vince la corsa sia in realtà il maratoneta più debole.

La prova di ciò sta nel fatto che quasi tutti gli studi di confronto condotti negli ultimi anni hanno fornito risultati di sostanziale parità. Parità che dipende sostanzialmente dal calo pressorio analogo, mentre eventuali effetti accessori dei farmaci non hanno il tempo di manifestarsi.

Lo studio LIFE [10] è l'unico in cui, in un tempo relativamente breve, sono emerse differenze nella capacità di prevenire gli eventi tra farmaci che abbiano prodotto un' eguale riduzione della PA. Tale studio ha evidenziato che, a parità di riduzione pressoria, $\mathrm{i}$ benefici erano maggiori in pazienti che ricevevano una terapia basata su un antagonista dell' angiotensina II (losartan) rispetto a coloro che ricevevano una terapia basata su un beta-bloccante. Se tutto ciò è emerso in un breve periodo di osservazione, dobbiamo supporre che tali differenze potrebbero essere persino più evidenti nel lungo e lunghissimo termine.

Altri parametri (ad esempio glicemia, colesterolemia, uricemia, etc.) dovrebbero essere sempre tenuti in conto. Nello studio ALLHAT, il profilo metabolico dei soggetti trattati con diuretico risultava peggiore di quelli trattati con altri farmaci, ma a ciò è stato dato poco rilievo perché tali alterazioni non sono esitate in eventi. Ciò è del tutto ovvio se si tiene conto della relativamente breve durata dello studio, ma è ragionevole pensare che aumenti di glicemia e colesterolo, nel corso degli anni, comportino necessariamente un incremento di complicanze.

Lo stesso studio LIFE ha mostrato come la comparsa di nuovi casi di diabete fosse minore nei pazienti trattati con l'antagonista

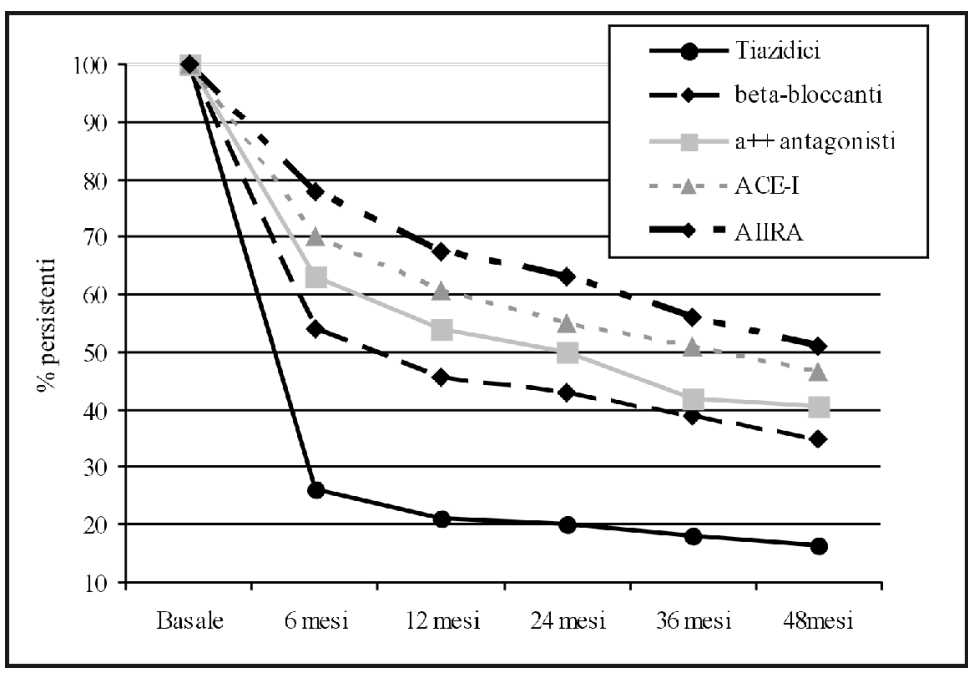

dell' angiotensina II rispetto a coloro che ricevevano il beta-bloccante. E il diabete, proprio per il sostanziale incremento di rischio che induce nei pazienti che ne sono affetti, è una patologia che produce un inevitabile incremento dei costi.

Negli anni più recenti si è, per fortuna, maggiormente analizzato l'impatto economico delle terapie antipertensive e le analisi economiche dello studio HOPE rappresentano un esempio di come, oltre agli aspetti clinici, anche le questioni economiche debbano contribuire alle scelte del medico. Dai risultati di tale studio si rileva che la terapia con un ACE-inibitore costa quanto il placebo (Tabella 2), mentre, se si calcolano i costi degli eventi risparmiati, produce un guadagno economico per il SSN e la società oltreché un guadagno in salute per i pazienti [11].

L'analisi ha infatti evidenziato che il costo di un anno di vita guadagnato con un trattamento a base di ACE-inibitore è pari a 1.940 euro, cifra che è circa la metà di quella ottenuta con una terapia a base di simvastatina nello studio $4 \mathrm{~S}$. È opportuno qui ricordare che viene generalmente considerato come economicamente vantaggioso un trattamento che produca un costo per anno di vita salvato non superiore ai 20.000 euro.

\section{Figura 4}

Persistenza al trattamento antipertensivo con differenti monoterapie [7] (PR Conlin et al, Clin Ther, 2001)

\section{Figura 5}

Persistenza al trattamento antipertensivo con differenti monoterapie. Pazienti seguiti presso il Centro perl'Ipertensione Arteriosa dell'Università degli Studi di Bologna (301 pazienti) (Costa FV data on file)

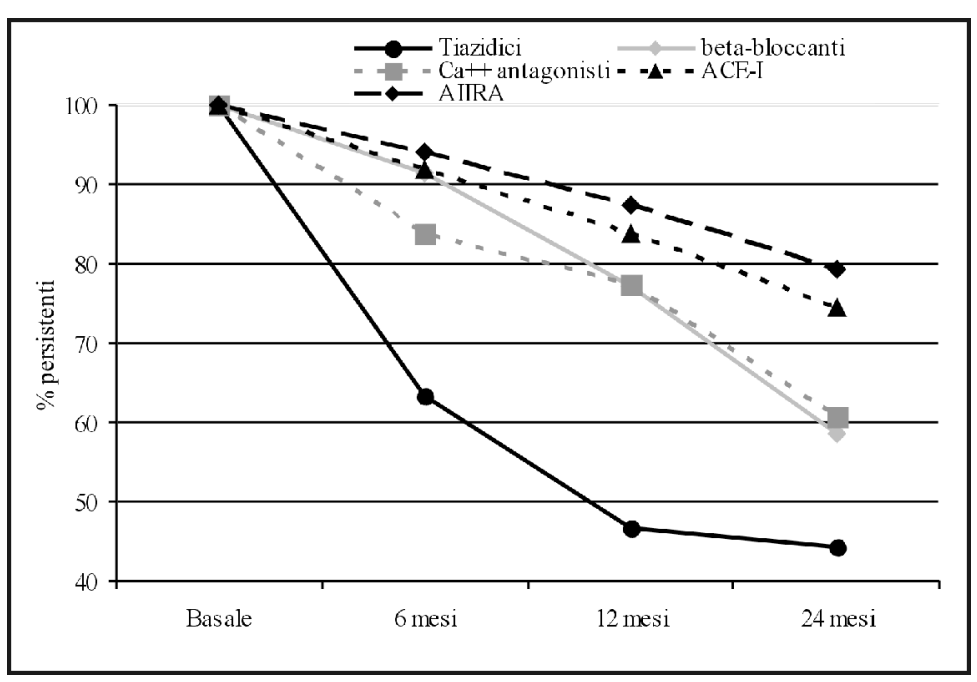


Una delle complicanze più costose dell'ipertensione e del diabete è l'insufficienza renale (dialisi, trapianto renale). In questo campo, il ruolo svolto dagli inibitori dell'angiotensina è fondamentale. Questi farmaci sono dotati di un effetto nefroprotettivo che pare prescindere dai loro effetti sulla pressione [12] e il fatto che molecole diverse abbiano prodotto effetti praticamente identici fa supporre che si tratti di un effetto di classe [13].

È stato calcolato [14], sulla base dei risultati dello studio RENAAL, che l'impiego di questi farmaci è in grado di produrre in quattro anni un risparmio netto di cinque miliardi e mezzo di euro e che l'entità del risparmio cresce all'aumentare degli anni di trattamento (oltre ai vantaggi per la vita dei pazienti). L'analisi dei dati ci evidenzia però che se l' analisi venisse limitata al primo anno di trattamento, tale vantaggio non emergerebbe.

Politici e amministratori sono abituati a pensare a bilanci annuali, e questo rappresenta uno dei principali ostacoli tra quanto è dimostrato scientificamente e la sua applicazione pratica.

L'insieme di questi studi clinici ci insegna che è necessario valutare costi e benefici in maniera corretta e che la terapia di patologie croniche, quali l'ipertensione, deve essere vista come un investimento a lungo termine e non semplicemente come un calcolo economico da effettuarsi in sei mesi o un anno.

Quando le valutazioni sono corrette e tengono conto di tutti i fattori coinvolti, anche farmaci dal prezzo elevato, come gli antagonisti dei recettori dell'angiotensina II, divengono assai convenienti dal punto di vista economico [15].

\section{OTTIMIZZAZIONE DEL COSTO/BENEFI- CIODIUNTRATTAMENTO}

Ottenere un miglioramento del rapporto costo-beneficio di un trattamento è possibile in diversi modi: riducendo i costi, aumentando i benefici o attuando entrambe le strategie. Il ruolo del medico in questa situazione è fondamentale.

Una diagnosi corretta è il primo passo ed evita di sottoporre a terapia pazienti che non ne necessitano, meccanismo questo che produce un incremento dei costi senza nessun beneficio.

Tabella 2

Costo medio per paziente per 4,5 anni in soggetti trattaticon ramipril e con placebo [11]
La riduzione dei prezzi dei farmaci è un problema politico, che non compete ai medici, ma deve essere comunque attentamente valutato nei suoi pro e contro. Se l'industria farmaceutica produce meno utili calano inevitabilmente anche le risorse destinate alla ricerca. Ciò avrebbe conseguenze gravi per lo sviluppo di nuovi farmaci e, in ultima analisi, per la salute dei pazienti.

La scelta del farmaco con cui iniziare il trattamento è un altro punto essenziale. Abbiamo visto come il prezzo d'acquisto non sia il solo parametro da valutare. Sono molto più importanti le garanzie che il paziente possa avere una buona compliance e proseguire nel tempo il trattamento senza modificarlo. Da questo punto di vista, gli ATII-antagonisti forniscono le garanzie di gran lunga migliori seguiti da ACE-inibitori e calcio-antagonisti.

Suggerire, come si tende ora a fare, di iniziare sempre la terapia con il diuretico, che secondo alcuni costerebbe di meno e sarebbe efficace come gli altri farmaci, è privo di fondamento per almeno due buone ragioni:

la stragrande maggioranza dei pazienti sospende di fatto il diuretico entro pochi mesi dall'inizio della terapia

quasi sempre, per ottenere un buon controllo della pressione, bisogna ricorrere a terapie d'associazione (spesso più di tre farmaci per paziente). Diventa, quindi, velleitario suggerire di usare farmaci a basso costo, quando nella realtà si dovrà far comunque ricorso a più farmaci associati.

Iniziare con un farmaco che non crei problemi al paziente e abbia ottime probabilità non solo di essere efficace, ma anche di garantire una buona compliance è un requisito essenziale per il successo di ogni terapia. In questo ambito, il diuretico continua a ricoprire un ruolo fondamentale, essendo il componente più frequentemente utilizzato nelle terapie di associazione. Le combinazioni precostituite tra basse dosi di diuretico tiazidico, ACE-inibitori e ATII-antagonisti, sono non solo estremamente efficaci, ma anche molto ben tollerate in quanto gli effetti avversi dei tiazidici vengono contrastati da questi farmaci. Oltretutto, il prezzo di queste associazioni è identico a quello del farmaco non associato, per cui è auspicabile inoltre che aumenti il numero disponibile di associazioni precostituite.

Un buon rapporto medico paziente è l'altro punto essenziale per il successo della terapia. Un aumento dei benefici è ottenibile infatti solo aumentando i soggetti in cui si ottiene un efficace controllo pressorio. Ciò è possibile migliorando compliance e persistenza, 
minimizzando il rischio di reazioni indesiderate e utilizzando schemi terapeutici che minimizzino il numero di assunzioni giornaliere. Tutti fattori in cui il ruolo del medico è primario.

Possibili ulteriori vantaggi sono ottenibili scegliendo terapie che correggano eventuali patologie associate. Esempi in tal senso sono forniti da antagonisti dell'angiotensina II e ACE-inibitori nei soggetti diabetici con e senza nefropatia, nei pazienti con ipertrofia ventricolare sinistra e nei pazienti con scompenso cardiaco. Parimenti utili possono essere gli alfa-bloccanti nei soggetti prostatici, o i beta-bloccanti negli anginosi e negli scompensati.

\section{Curare i pazienti ipertesi}

\section{è un costo o un guadagno per la società?}

Ovviamente, curare al meglio tutti i pazienti è per il medico un obbligo deontologico prioritario rispetto ad ogni considerazione economica. Ma la domanda posta può anche essere intesa come semplice quesito economico.

I dati disponibili ci consentono di affermare che curare bene l'ipertensione significherebbe anche risparmiare risorse economiche. L'ipertensione infatti è una patologia che, se non curata, causa complicanze non solo gravi clinicamente, ma anche assai costose per la società e gli individui.

Uno studio recente ha evidenziato come in Italia, nella situazione attuale di controllo dell'ipertensione, gli eventi acuti cardiovascolari siano molto frequenti e come essi potrebbero essere drasticamente ridotti se si raggiungessero valori pressori normali nella totalità dei pazienti (Figura 6) [16]. Questo tipo di calcolo è stato fatto sulla base dei risultati ottenuti dallo Studio HOT.

È stato anche calcolato quale sarebbe l'impatto economico di tale intervento aggressivo ed estensivo (Figura 7): solo riducendo i costi relativi alla fase acuta degli eventi (infarto, scompenso cardiaco ed ictus), la spesa scenderebbe da 2.045 a 263 milioni di euro per anno.

Ora, se si considera che circa due terzi dei soggetti colpiti da ictus sopravvivono all'episodio acuto e che la gestione dei sopravvissuti è molto costosa (da 20.000 a oltre 100.000 euro/anno a seconda dei casi e delle strutture in cui i pazienti vengono seguiti), si possono tentare alcuni calcoli (Tabella 3).

Sulla base dei dati di spesa per paziente per anno e di spesa farmaceutica totale annua per i farmaci antipertensivi (circa 1,5-1,7 miliardi di euro nel luglio 2003) possiamo valutare che oggi, in Italia, non più di 3-4 milioni di ipertesi ricevono una qualche terapia.

Alla luce dei dati epidemiologici più recenti, i pazienti ipertesi in Italia non sono meno di 15 milioni. Se pensassimo di trattarli tutti, sulla base

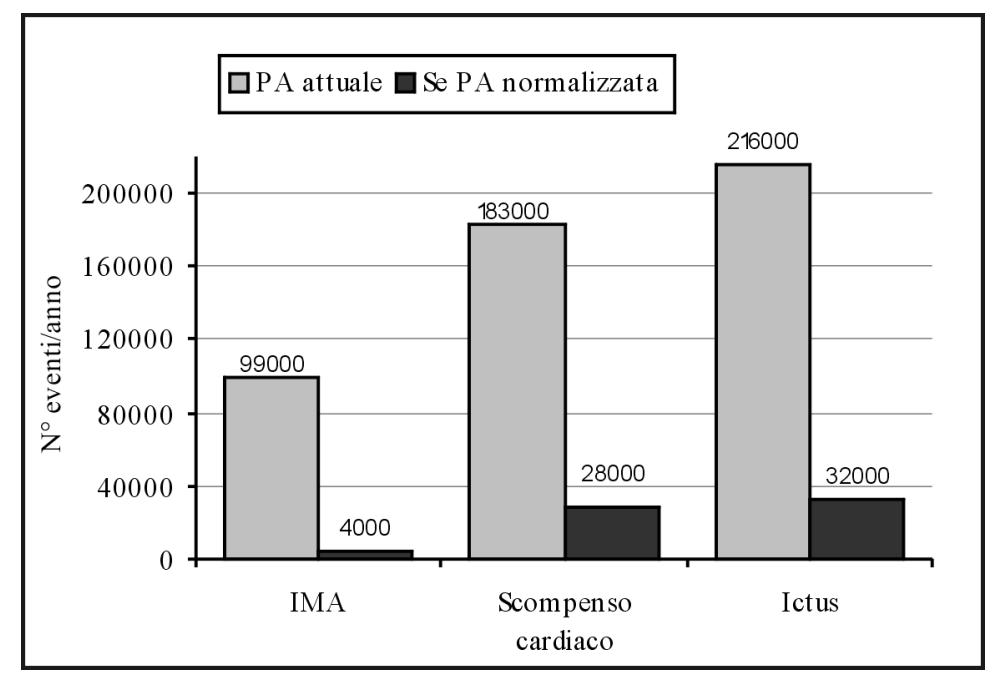

di quanto spendiamo oggi per ciascuno di essi (circa 500 euro/anno), dovremmo spendere circa 7,2 miliardi di euro per anno. Se li trattassimo in maniera aggressiva, spendendo il doppio, la cifra salirebbe a 14,4 miliardi di euro/anno.

Attualmente, solo per gli ictus, spendiamo da un minimo di 6,5 ad un massimo di 16,2 miliardi di euro ogni anno. Tale cifra scenderebbe a 1,0-2,9 miliardi di euro se la pressione di questi pazienti venisse normalizzata.

Si può così vedere come i costi di una terapia estesa a tutti e di intensità doppia verrebbero già pareggiati considerando solo la riduzione dell'ictus. E da tale calcolo sono escluse altre complicanze gravi e costose come l'infarto miocardico, lo scompenso cardiaco, l'insufficienza renale. Se anche questo tipo di complicanze venisse incluso nel conto, il vantaggio economico di un trattamento esteso e intensivo diverrebbe ancora più evidente.

È ovvio, che alla luce di queste considerazioni, il costo di una monoterapia rispetto ad un altra diventa irrilevante dato che, per raggiungere gli obiettivi pressori ottimali, si deve ricorrere sempre a terapie d'associazione.

All'interno di queste ultime convivono necessariamente molecole ad alto e a basso costo. Non si tratta quindi di demonizzare i vec-

\section{Figura 6}

Eventi cardiovascolari acuti in Italia in relazione al controllo pressorio [16]

\section{Figura 7}

Costo annuo degli eventi cardiovascolari acuti in Italia in relazione al controllo pressorio [16]

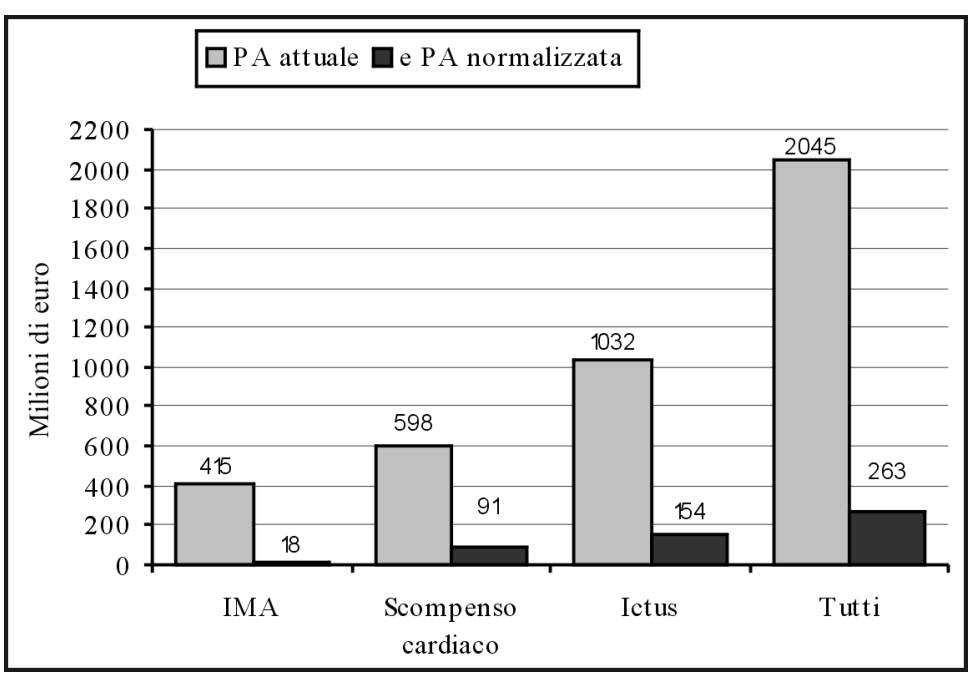




\begin{tabular}{lccc}
\hline & $\begin{array}{c}\text { Attuale } \\
(\mathbf{2 0 0 2})\end{array}$ & Se la PA < 140/90 & Differenza \\
& 216.00 & 32.000 & -184.000 \\
\hline $\mathrm{N}^{\circ}$ ictus/anno & 72.000 & 11.000 & -61.000 \\
fatali & 144.000 & 21.000 & -123.000 \\
non fatali & $6.5-16.2$ & $1.0-2.9$ & $-5.5-13.3$ \\
Costo totale ictus & & $1.5-1,7$ \\
$\begin{array}{l}\text { Costo attuale Terapia } \\
\text { (3 milioni di pazienti) (miliardi euro/anno) }\end{array}$ \\
$\begin{array}{l}\text { Costo se si rattassero } 15 \text { milioni di ipertesi } \\
\text { (miliardi euro/anno) }\end{array}$
\end{tabular}

\section{Tabella 3}

Prevalenza attuale dell'ictus in Italia $e$ suoi costi e impatto della normalizzazione dei valori pressori [da 16] chi farmaci quali i diuretici, che restano uno dei cardini irrinunciabili della terapia, ma di sottolineare che il loro utilizzo deve essere attuato in modo da far sì che i pazienti siano in grado di assumerli senza problemi.

Ciò diventa possibile quando i diuretici vengono somministrati a dosi basse, in associazione a farmaci come gli antagonisti dell'angiotensina II e gli ACE-inibitori, che ne contrastano gli effetti metabolici avversi, e dopo che la terapia è stata avviata con le molecole più moderne, che garantiscono nel tempo i massimi livelli di persistenza.

La terapia antipertensiva deve, quindi, essere vista non come una gara tra singoli indivi- dui, ma come una competizione a squadre in cui ogni farmaco gioca un ruolo importante e diverso.

Si deve, inoltre, cambiare radicalmente mentalità e passare da una visione che ritiene la terapia antipertensiva solo un costo a quella che considera l'ipertensione come una malattia che produce complicanze e costi evitabili, sicché la sua terapia è un investimento vantaggioso che a lungo termine può ridurre gli attuali costi sanitari e potrebbe addirittura indurre risparmi netti per la società.

Il costo per anno di vita guadagnato o per QALY aggiuntivo, stimato per le moderne terapie antipertensive basate sull'uso di farmaci capaci di prolungare la vita, ridurre le complicanze cardiovascolari e migliorare la qualità di vita, è paragonabile o inferiore a quelli delle statine e nettamente inferiore al tetto massimo convenzionalmente ritenuto oggi ammissibile dai paesi industrializzati dell'occidente.

Si può quindi concludere che la terapia antipertensiva moderna, a fronte di un incremento della spesa farmaceutica, riduce significativamente gli altri costi sanitari e i costi indiretti correlabili all'ipertensione, aumenta il numero di anni di vita guadagnati dalla popolazione e migliora complessivamente la qualità di vita dei pazienti e dei loro famigliari.

\section{BIBLIOGRAFIA}

1) Wolf-Maier K,Cooper RS, Banegas JR et al. Hypertension prevalence and blood pressure levels in 6 European countries, Canada and the United States. Jama,2003;289:2363-2369

2) Dormi A, Gaddi A. Brisighella Heart Study, data on file

3) Hilleman DE, Mohiuddin SM, Lucas BD Je et al. Cost-minimization analysis of initial antihypertensive therapy in patients with mild-to-moderate essential diastolic hypertension. Clin Ther 1994;16:88-102.

4) Costa FV. Assessment of cost-benefit in antihypertensive treatment. In "Manual of hypertension" edited by G.Mancia et al, Churchill Livingston: 673-684,2002.

5) Jones JK et al. Discontinuation of and changes in treatment after start of new courses of antihypertensive drugs: a study of United Kingdom population. Br Med J, 1996; 311:293-295

6) The ALLHAT Officers and Co-ordinators for the ALLHAT Collaborative Group. Major outcomes in high-risk hypertensive patients randomized to angiotensin converting enzyme inhibitor or calcium channel blocker vs. diuretic. The Antihypertensive and LipidLowering Treatment to Prevent Heart Attack Trial (ALLHAT). Jama,2003;288:1981-1997.

7) Conlin PR, Gerth WC, Fox J et al. Four-year persistence patterns among patients initiating therapy with the angiotensin II receptor antagonist losartan versus other antihypertensive classes. Clin Ther,2001;12:1999-2010.

8) Degli Esposti E, Sturani A, Di Martino M et al. Long-term persistence with antihypertensive drugs in new patients. J Human Hypertens, 2002;16:439-444

9) Ambrosioni E, Costa FV. Cost-effectiveness calculations from trials. J Hypertension ,1996:47s-52s.

10) Dahlof B, Devereux RB, Kjeldsen SE et al. Cardiovascular morbidity and mortality in the Losartan Intervention For Endpoint reduction in hypertension study (LIFE): a randomized trial against atenolol. Lancet. 2002,359:995-1003.

11) Lamy A, Yusuf S, Pogue J et al. Cost implications of the use of ramipril in high-risk patients based on the Heart Outcomes Prevention Evaluation (HOPE) study. Circulation.2003;107:960-965.

12) Epstein M. Recent landmark clinical trials: how do they modify the therapeutic paradigms? Am J Hypertens.2002;82s-84s.

13) Anonymous editorial. Class benefit of ATl antagonists in Type 2 diabetes with nephropathy. Expert Opin Pharmacother.2002;3:625-628

14) Herman WH, Shaninfar S, Carides GW et al. Losartan reduces the cost associated with diabetic end-stage renal disease. The RENAAL study economic evaluation. Diabetes Care.2003;26:683-687.

15) McIntyre H, Costa FV, Dusing R et al. The role of losartan in cost effective hypertension control. Curr Med Res Opini. 2002;18:139-145.

16) Hansson L, Lloyd A, Anderson P, Kopp Z. Excess morbidity and cost of failure to achieve target for blood pressure control in Europe. Blood press.2002;11:35-45. 